\title{
Optimal Neuronal Tuning for Finite Stimulus Spaces
}

\author{
W. Michael Brown \\ wmbrown@sandia.gov \\ Computational Biology, Sandia National Laboratories, Albuquerque, NM, 87123, \\ U.S.A.
}

\begin{abstract}
Alex Bäcker
alex@caltech.edu

Computational Biology, Sandia National Laboratories, Albuquerque, NM, 87123, and

Division of Biology, California Institute of Technology, Pasadena, CA 91125, U.S.A.
\end{abstract}

The efficiency of neuronal encoding in sensory and motor systems has been proposed as a first principle governing response properties within the central nervous system. We present a continuation of a theoretical study presented by Zhang and Sejnowski, where the influence of neuronal tuning properties on encoding accuracy is analyzed using information theory. When a finite stimulus space is considered, we show that the encoding accuracy improves with narrow tuning for one- and twodimensional stimuli. For three dimensions and higher, there is an optimal tuning width.

\section{Introduction}

The potential impact of coding efficiency on neuronal response properties within the central nervous system was first proposed by Attneave (1954) and has since been studied using both theoretical and experimental approaches. The issue of optimal neuronal tuning widths has received much attention in recent literature. Empirical examples of both finely tuned receptive fields (Kuffler, 1953; Lee, 1999) and broadly tuned neurons (Georgopoulos, Schwartz, \& Kettner, 1986; Knudsen \& Konishi, 1978) have been found. Theoretical arguments have also been made for both sharp (Barlow, 1972; Lettvin, Maturana, McCulloch, \& Pitts, 1959) and broad (Baldi \& Heiligenberg, 1988; Eurich \& Schwegler, 1997; Georgopoulos et al., 1986; Hinton, McClelland, \& Rumelhart, 1986; Salinas \& Abbott, 1994; Seung \& Sompolinsky, 1993; Snippe, 1996; Snippe \& Koenderink, 1992) tuning curves as a means to increase encoding accuracy.

Using Fisher information, Zhang and Sejnowski (1999) offered an intriguing solution where the choice of narrow or broad tuning curves depends on the dimensionality of the stimulus space. They found that for one dimension, the encoding accuracy increases with decreasing tuning 
width and that for two dimensions, the encoding accuracy is independent of the tuning width. For three dimensions and higher, the results suggest that encoding accuracy should increase with increasing tuning width. The result, which is widely cited in works on neuronal encoding, offers a universal scaling rule for all radial symmetric tuning functions. However, this scaling rule is highly unintuitive in that for greater than three dimensions, it predicts optimal encoding accuracy for infinite tuning widths, that is, tuning widths for which neurons have no discrimination power and all neurons are indistinguishable from each other. In this note, we analyze this effect and show that when a finite stimulus space is considered, there is an optimal tuning width (in terms of Fisher information) for all stimulus dimensionalities.

\section{Fisher Information}

2.1 Fisher Information for an Infinite Stimulus Space. The CramérRao inequality gives a lower bound for the variance of any unbiased estimator (Cover \& Thomas, 1991) and is useful for studying neuronal encoding accuracy in that it represents the minimum mean-squared reconstruction error that can be achieved by any decoding strategy (Seung \& Sompolinsky, 1993). Let $\mathbf{x}=\left(x_{1}, x_{2}, \ldots, x_{D}\right)$ be a vector describing a $D$-dimensional stimulus. The Cramér-Rao bound is then given by

$$
v(\mathbf{x}) \geq J^{-1}(\mathbf{x})
$$

where $v$ is the covariance matrix of a set of unbiased estimators for $\mathbf{x}, J$ is the Fisher information matrix, and the matrix inequality is given in the sense that $v(\mathbf{x})-J^{-1}(\mathbf{x})$ must be a nonnegative definite matrix (Cover \& Thomas, 1991). For an encoding variable representing neuronal firing rates, the Fisher information matrix for a neuron is given by

$$
J_{i j}(\mathbf{x})=E\left[\left(\frac{\partial}{\partial x_{i}} \ln P[\mathbf{n} \mid \mathbf{x}, \tau]\right)\left(\frac{\partial}{\partial x_{j}} \ln P[\mathbf{n} \mid \mathbf{x}, \tau]\right)\right],
$$

where $E$ represents the expectation value over the probability distribution $P[\mathbf{n} \mid \mathbf{x}, \tau]$ for firing $\mathbf{n}$ spikes at stimulus $\mathbf{x}$ within a time window $\boldsymbol{\tau}$. For multiple neurons with independent spiking, the total Fisher information for $N$ neurons is given by the sum

$$
J(\mathbf{x})=\sum_{a=1}^{N} J_{a}(\mathbf{x})
$$

If the neurons are restricted to radial symmetric tuning functions and distributed identically throughout the stimulus space such that the 
distributions of estimation errors in each dimension are identical and uncorrelated, the Fisher information matrix becomes diagonal (Zhang \& Sejnowski, 1999), and the total Fisher information reduces to

$$
J(\mathbf{x})=\sum_{a=1}^{N} \sum_{i=1}^{D} E\left(\frac{\partial}{\partial x_{i}} \ln P_{a}[\mathbf{n} \mid \mathbf{x}, \tau]\right)^{2}
$$

For homogeneous Poisson spike statistics,

$$
P[n \mid \mathbf{x}, \tau]=\frac{(\tau \cdot f(\mathbf{x}))^{n}}{n !} \exp (-\tau f(\mathbf{x}))
$$

where $f(\mathbf{x})$ describes the mean firing rate of the neuron with respect to the stimulus, or the neuronal tuning function. Equation 2.4 then becomes

$$
J(\mathbf{x})=\tau \sum_{a=1}^{N} \sum_{i=1}^{D}\left(\frac{\partial}{\partial x_{i}} f_{a}(\mathbf{x})\right)^{2} \frac{1}{f_{a}(\mathbf{x})}
$$

For a gaussian tuning function,

$$
f(\mathbf{x})=F \exp \left(-\frac{1}{2 \sigma^{2}} \sum_{i=1}^{D}\left(x_{i}-c_{i}\right)^{2}\right)
$$

where $F$ represents the mean peak firing rate, $\mathbf{c}=\left(c_{1}, c_{2}, \ldots, c_{D}\right)$ represents the preferred stimulus of the neuron, and $\sigma$ represents the tuning width parameter for the neuron. Substitution into equation 2.6 gives

$$
J(\mathbf{x})=\tau \sum_{a=1}^{N} \frac{1}{\sigma^{4}}\left[\sum_{i=1}^{D}\left(x_{i}-c_{a, i}\right)^{2}\right] \exp \left(-\frac{1}{2 \sigma^{2}} \sum_{i=1}^{D}\left(x_{i}-c_{a, i}\right)^{2}\right)
$$

Assuming that the preferred stimuli for the neurons are uniformly distributed throughout the stimulus space, the average Fisher information per neuron for an infinite stimulus space can be found by replacing the summation with an integral (Zhang \& Sejnowski, 1999):

$$
\bar{J}=\int_{-\infty}^{\infty} J_{1}(\mathbf{x}) d x_{1}, \ldots, d x_{D}
$$


where $J_{1}(\mathbf{x})$ represents the Fisher information for a single neuron rather than the total as given in equation 2.4. Under these assumptions, we can make the replacement:

$$
\begin{gathered}
\boldsymbol{\xi}=\mathbf{x}-\mathbf{c} \\
\bar{J}=\int_{-\infty}^{\infty} J_{1}(\xi) d \xi_{1}, \ldots, d \xi_{D}
\end{gathered}
$$

For Poisson spike statistics and gaussian tuning (see equation 2.8),

$$
\bar{J}=\frac{F \tau}{\sigma^{4}}\left[-\frac{\sigma^{2}}{2}\left(2 \xi \exp \left(-\frac{\xi^{2}}{2 \sigma^{2}}\right)-\sigma \sqrt{2 \pi} \cdot \operatorname{erf}\left(\frac{\xi \sqrt{2}}{2 \sigma}\right)\right)\right]_{-\infty}^{\infty}
$$

for $D=1$. Here, the gaussian error function is given by

$$
\operatorname{erf}(b)=\frac{2}{\sqrt{\pi}} \int_{0}^{b} \exp \left(-t^{2}\right) d t
$$

If we assume that the stimulus space (integration interval) is infinite and that $\sigma$ is finite, equation 2.11 reduces to

$$
\overline{J_{1 D}}=\frac{F \tau \sqrt{2 \pi}}{\sigma}
$$

Due to symmetry with respect to different dimensions, equation 2.13 can be generalized to any dimensionality to give the result reported by Zhang and Sejnowski (1999):

$$
\bar{J}=F d \tau(2 \pi)^{d / 2} \sigma^{d-2}
$$

The Fisher information based on equation 2.14 as a function of tuning width is shown in Figure 1. Although we have shown the Fisher information per neuron as an average, it is actually the exact Fisher information for each neuron because of the assumption of homogeneous tuning widths and an infinite stimulus space. Therefore, the total Fisher information can be found by multiplying the Fisher information per neuron by the number of neurons to get Fisher information across the stimulus space that is independent of $\mathbf{x}$. Equation 2.14 does not describe the influence of tuning width on encoding accuracy in the limit as $\sigma$ approaches infinity, however, and therefore is unusable when the tuning width is large relative to the stimulus space. This is relevant, since for $\mathrm{D}>2$, equation 2.14 predicts optimal tuning widths to be infinite. Furthermore, when using neuronal firing rate as an encoding variable, this becomes relevant in that for a finite number of neurons with 


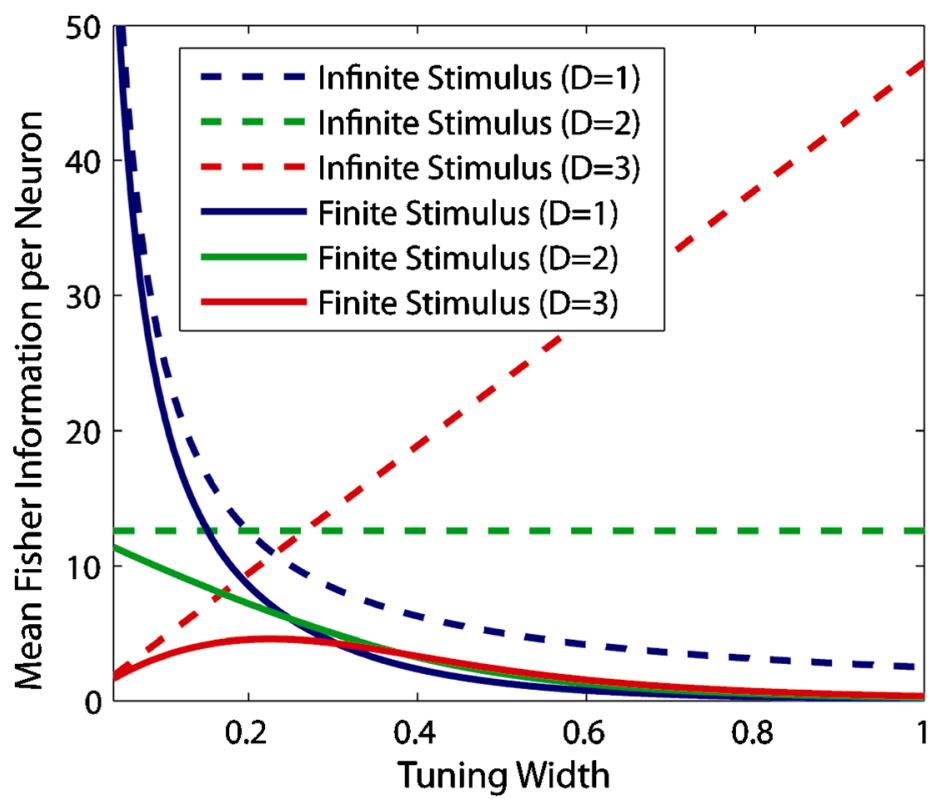

Figure 1: Scaling rule for the average Fisher information per neuron as a function of tuning width for different stimulus dimensionalities. Dashed lines represent the Fisher information calculated when the stimulus space is infinite (see equation 2.14), and solid lines represent calculations for a finite stimulus space (see equation 2.15). In this plot, the Fisher information is divided by the peak firing rate $(F)$ and the time window $(\tau)$.

finite firing rates and a finite decoding time, the range for the stimulus must be finite. That is, with discrete spiking events, there is a range of stimulus space beyond which no Fisher information can be conveyed within a reasonable decoding time.

2.2 Fisher Information for a Finite Stimulus Space. In order to study Fisher information within a finite stimulus space, we begin by considering a stimulus range normalized to lie in the inclusive range between 0 and 1. In this case, the tuning width is expressed in terms of a fraction of the finite stimulus space. If we consider an infinite number of neurons with preferred stimuli evenly distributed across the finite stimulus space, the average Fisher information per neuron is given (for radial symmetric tuning functions) by

$$
\overline{J_{f}}=\int_{0}^{1} d c_{1}, \ldots, d c_{D} \int_{0}^{1} J_{1}(\mathbf{x}, \mathbf{c}) d x_{1}, \ldots, d x_{D}
$$


where $J_{1}(\mathbf{x}, \mathbf{c})$ is the Fisher information at $\mathbf{x}$ for the neuron with preferred stimulus c. Here,

$$
J_{1}(\mathbf{x}, \mathbf{c})=\tau \frac{1}{\sigma^{4}}\left[\sum_{i=1}^{D}\left(x_{i}-c_{i}\right)^{2}\right] \exp \left(-\frac{1}{2 \sigma^{2}} \sum_{i=1}^{D}\left(x_{i}-c_{i}\right)^{2}\right) .
$$

For the one-dimensional case with Poisson spiking statistics and gaussian tuning (see equation 2.8), the average Fisher information per neuron is given by

$$
\overline{J_{f 1 D}}=F \tau\left(\frac{\sqrt{2 \pi}}{\sigma} \operatorname{erf}\left(\frac{\sqrt{2}}{2 \sigma}\right)+4 \exp \left(-\frac{1}{2 \sigma^{2}}\right)-4\right),
$$

for the two-dimensional case by

$$
\overline{J_{f 2 D}}=4 F \tau\left(\begin{array}{l}
\pi\left(\operatorname{erf}\left(\frac{\sqrt{2}}{2 \sigma}\right)\right)^{2}+3 \sigma \sqrt{2 \pi} \operatorname{erf}\left(\frac{\sqrt{2}}{2 \sigma}\right)\left(\exp \left(\frac{-1}{2 \sigma^{2}}\right)-1\right) \\
+4 \sigma^{2} \exp \left(\frac{-1}{\sigma^{2}}\right)\left(1-2 \exp \left(\frac{1}{2 \sigma^{2}}\right)\right)+4 \sigma^{2}
\end{array}\right),
$$

and for the three-dimensional case by

$$
\begin{aligned}
& \overline{J_{f 3 D}}= \\
& 12 \sigma F \tau\left(\begin{array}{l}
\pi^{3 / 2} \frac{\sqrt{2}}{2}\left(\operatorname{erf}\left(\frac{\sqrt{2}}{2 \sigma}\right)\right)^{3}-4 \sigma \pi\left(\operatorname{erf}\left(\frac{\sqrt{2}}{2 \sigma}\right)\right)^{2}\left(1-\exp \left(\frac{-1}{2 \sigma^{2}}\right)\right) \\
-5 \sigma^{2} \sqrt{2 \pi} \operatorname{erf}\left(\frac{\sqrt{2}}{2 \sigma}\right)\left(2 \exp \left(\frac{-1}{2 \sigma^{2}}\right)-\exp \left(\frac{-1}{\sigma^{2}}\right)-1\right) \\
+4 \sigma^{3}\left(3 \exp \left(\frac{-1}{2 \sigma^{2}}\right)-3 \exp \left(\frac{-1}{\sigma^{2}}\right)+\exp \left(\frac{-3}{2 \sigma^{2}}\right)-1\right)
\end{array}\right) .
\end{aligned}
$$

The influence of tuning width on the average Fisher information per neuron is plotted in Figure 1 for the first $\mathrm{D}=1-3$ dimensionalities. For one and two dimensions, the average Fisher information, and thus encoding accuracy, increases with decreasing tuning width. For three dimensions, there is an optimal tuning width in terms of Fisher information, given by the $\sigma$ at which $\mathrm{d} J / \mathrm{d} \sigma=0$ (approximately $22.541 \%$ of the stimulus space).

For higher dimensionalities, optimal tuning widths also exist. This can be seen by the fact that as the tuning width approaches infinity, the derivative of the probability of firing a given number of spikes with respect to a 
stimulus goes to zero. For the example presented here,

$$
\lim _{\sigma \rightarrow \infty} \frac{\partial}{\partial x_{k}} f(\mathbf{x})=\lim _{\sigma \rightarrow \infty} \frac{F\left(c_{k}-x_{k}\right)}{\sigma^{2}} \exp \left(-\frac{1}{2 \sigma^{2}} \sum_{i=1}^{D}\left(x_{i}-c_{i}\right)^{2}\right)=0
$$

for any $k$ from 1 to $D$. In this limit, the tuning function is independent of $\mathbf{x}$, the probability distribution for spike firing (see equation 2.5 ) is independent of $\mathbf{x}$, and the resulting derivatives give a Fisher information of zero (see equation 2.2). In the limit as the tuning width goes to zero, equation 2.14 becomes valid (the limits of the error functions and exponentials are equivalent in both the case where the tuning width is infinitesimal and the case when the stimulus space is infinite):

$$
\begin{aligned}
\lim _{\sigma \rightarrow 0} \overline{J_{f}} & =\lim _{\sigma \rightarrow 0} \int_{0}^{1} d c_{1}, \ldots, d c_{D} \int_{0}^{1} J_{1}(\mathbf{x}, \mathbf{c}) d x_{1}, \ldots, d x_{D} \\
& =\lim _{\sigma \rightarrow 0} \int_{-\infty}^{\infty} J_{1}(\boldsymbol{\xi}) d \xi_{1}, \ldots, d \xi_{D} \\
& =\left\{\begin{array}{l}
\infty, D=1 \\
2 F d \tau \pi, D=2 \\
0, D>2 .
\end{array}\right.
\end{aligned}
$$

Therefore, at least one maximum in the Fisher information must exist for higher dimensionalities. While we expect that the optimal tuning width shifts toward a larger fraction of the stimulus space as the dimensionality is increased, we are unable to find a closed form for equation 2.15 that can prove this.

The deviation of the results from equation 2.14 is explained by the fact that an increase in tuning width in equation 2.15 results in an increase in tuning width relative to the stimulus space, an effect that is not easily seen when an infinite stimulus space is considered. Clearly, this deviation should be expected, as the limit given in equation 2.20 contradicts the result in equation 2.14. An infinite tuning width produces a neuronal tuning function that is flat at all but infinite stimuli. A neuron with such a tuning curve cannot discriminate between finite stimuli and therefore contributes zero Fisher information within finite stimulus ranges. This result, which is true for all dimensionalities, tells us that an infinite tuning width can never be optimal, at least under the assumptions presented in this work.

2.3 Determining the Finite Stimulus Range. As stated earlier, when using neuronal firing rate as an encoding variable, there is a physiological limit to the range of stimulus space that can be perceived. This limit is due 


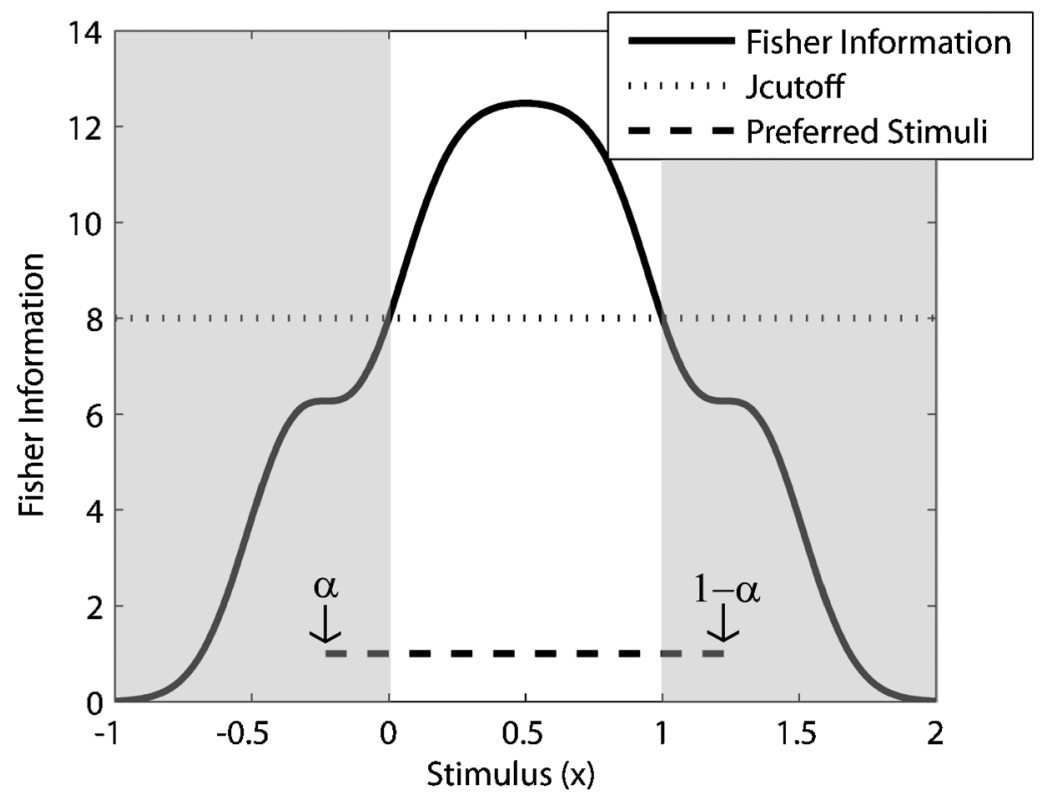

Figure 2: Fisher information as a function of stimulus $\left(J_{\mathbf{x}}(\mathbf{x})\right)$ calculated using equation 2.24. The average Fisher information per neuron calculated using equation 2.22 is found by normalizing the finite stimulus space to lie between 0 and 1 and distributing the preferred stimuli of the neurons between $\alpha$ and $1-\alpha$ such that the drop in Fisher information at the corners of the stimulus space does not fall below $J_{\text {cutoff. }}$ The shaded regions are not included in the finite stimulus space and therefore are not included in the average. For this plot, $\sigma=0.2, J_{\text {cutoff }}=8$, and $\alpha=-0.23$. The Fisher information is divided by the peak firing rate $(F)$ and the time window $(\tau)$.

to the fact that the number of neurons is finite, the firing rates are finite, and the decoding time is finite. This limit will depend on the number of neurons, the preferred stimuli of the neurons, the mean peak firing rate, and the decoding time. There is no implication, however, that the stimulus space range should equal the range of preferred stimuli for the neurons. It is therefore important to consider the influence of the preferred stimuli range on the optimal tuning width.

We can evaluate the effect of the preferred stimuli range on the optimum tuning width by fixing the integration interval for the stimulus space $(\mathbf{x})$ to lie between 0 and 1 , and setting the integration interval for the preferred stimuli (c) such that the drop in Fisher information at the corners of the stimulus space does not fall below some threshold value $\left(J_{\text {cutoff }}\right)$ (see Figure 2 ). In this case, the tuning width always represents a fraction of the stimulus space. The range of the preferred stimuli is dependent on both $\sigma$ and $J_{\text {cutoff; }}$; 
however, it will always be centered on the stimulus space and identical in each dimension. The choice of the Fisher information as a threshold to limit the stimulus space is reasonable because there is a finite range of stimulus space within which the mean-squared error will be tolerable based on a finite distribution of preferred stimuli.

We can evaluate the effect of $J_{\text {cutoff }}$ on the Fisher information per neuron in terms of $\sigma$ as

$$
\overline{J_{\alpha}}(\sigma, \alpha)=\frac{1}{(1-2 \alpha)^{D}} \int_{\alpha}^{1-\alpha} d c_{1}, \ldots, d c_{D} \int_{0}^{1} J_{1}(\mathbf{x}, \mathbf{c}) d x_{1}, \ldots, d x_{D},
$$

such that

$$
J_{\mathbf{x}}\left(x_{1}=0, \ldots, x_{D}=0\right)=J_{\mathbf{x}}\left(x_{1}=1, \ldots, x_{D}=1\right)=J_{\text {cutoff }}
$$

where $J_{\mathbf{x}}$ represents the Fisher information as a function of the stimulus

$$
J_{\mathbf{x}}(\mathbf{x})=\int_{\alpha}^{1-\alpha} J_{1}(\mathbf{x}, \mathbf{c}) d c_{1}, \ldots, d c_{D}
$$

and $\alpha$ determines the range of the preferred stimuli. For a given $J_{\text {cutoff }}$ and $\sigma$, we solve for the range of the preferred stimuli $(\alpha-1-\alpha)$ using a numerical evaluation of equation 2.23 such that the Fisher information at the corners of the stimulus space will be equal to $J_{\text {cutoff. }}$. Of course, for each value of $\sigma$, there is a maximum $J_{\text {cutoff }}$ for which a solution exists simply because the Fisher information at any point in the stimulus space is finite. Once $\alpha$ has been determined, an analytic solution to equation 2.22 can then be solved to determine the average Fisher information per neuron over the stimulus space range, using a range of preferred stimuli, which may be larger or smaller than the range of the stimulus space, depending on the value of $J_{\text {cutoff. }}$. Because equation 2.22 represents an average Fisher information per neuron, it is normalized to account for the range of the neuronal distribution.

For a finite neuronal distribution range, there is a finite range of stimulus space within which the mean squared error will be tolerable. By determining the average Fisher information per neuron using equation 2.22, the finite stimulus space is always determined by the points at which the Fisher information begins to fall below this threshold, regardless of the tuning width. The results for this evaluation are shown in Figure 3 for the first three dimensionalities. For all cases, an increase in $J_{\text {cutoff }}$ results in an increase in the range of the preferred stimuli (decrease in $\alpha$ ); that is, when the cutoff becomes low, the range of the stimulus space must become larger relative to the distribution of the neurons such that the stimulus space includes 

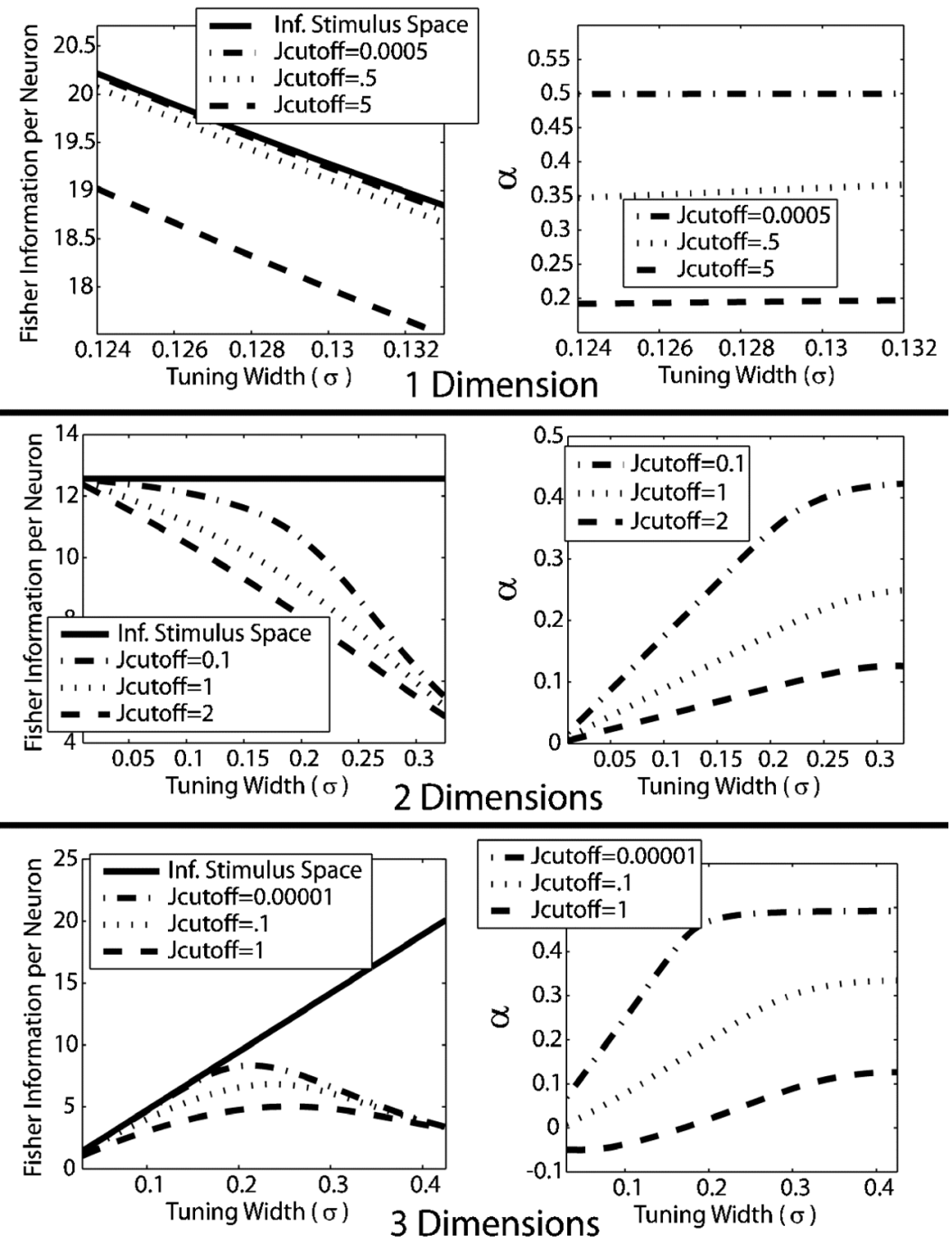

Figure 3: Plot of the average Fisher information per neuron for one to three dimensions calculated using equation 2.22 (left) along with the corresponding ranges for the preferred stimuli (right). The plotted values for $J_{\text {cutoff }}$ were chosen so that the range of $\sigma$ is large enough to illustrate differences (as $\sigma$ increases, the maximum $J_{\text {cutoff }}$ that can be achieved decreases). For all dimensionalities, there is an increase in the range of preferred stimuli and a decrease in the average Fisher information per neuron as $J_{\text {cutoff }}$ increases. The Fisher information is divided by the peak firing rate $(F)$ and the time window $(\tau)$. 
regions with higher mean squared errors. This increase in the range of the preferred stimuli results in a decrease in the average Fisher information per neuron. This is due to the fact that the total Fisher information within the finite stimulus space (0-1) for a single neuron decreases as c deviates from 0.5 . As the range of the preferred stimuli increases, the average Fisher information for each neuron within the stimulus space will decrease.

The results quantitate the idea that the encoding accuracy within a finite stimulus space of interest can be increased by increasing the range of preferred stimuli around the center of the stimulus space. This increase comes at an energetic cost resulting from an increase in the number of neurons, with diminishing returns due to a decrease in the average Fisher information per neuron within the stimulus space. While a change in the curvature of the average Fisher information as a function of $\sigma$ can result from a change in the Fisher information cutoff, the Fisher information per neuron always improves with narrow tuning for one and two dimensions. For three dimensions, there will always be an optimal tuning width; however, as shown in Figure 3, the optimal tuning width will shift toward a larger fraction of the stimulus space with an increase in the Fisher information cutoff. Numerical evaluation of the optimal tuning width calculated with $J_{\text {cutoff }}$ ranging from $10^{-5}$ to 10 results in an increase in the optimal tuning width from 0.21 to 0.41 , a decrease in $\alpha$ from 0.48 to -0.5 , and a decrease in the optimal average Fisher information per neuron from 8.34 to 1.78 .

\section{Conclusion}

The result in equation 2.14 gives the Fisher information per neuron when an infinite stimulus space is considered. It is implicit in this model that the Fisher information at any point in the stimulus space is constant (independent of the stimulus) due to an infinite range of the preferred stimuli for the neurons (see the substitution in equation 2.10). We have continued this analysis using a finite stimulus space for two reasons. First, equation 2.14 is not accurate when the tuning width is large relative to the stimulus space due to an assumption in the derivation that $\sigma$ is finite. Therefore, it is convenient to use a finite stimulus space in order to ascertain accurate results at any $\sigma$. Second, physiological limits preclude both the case where the stimulus space is infinite and the case where the range of the preferred stimuli is infinite. Using a finite stimulus space and a finite range of preferred stimuli introduces edge effects that are important to consider in models of encoding accuracy simply because these edge effects must exist in animal physiology.

In the case where a finite range of preferred stimuli is considered, the Fisher information cannot be independent of the stimulus, even when an infinite number of neurons are considered. This results in limited regions of the stimulus space where the encoding accuracy is tolerable. When using a finite stimulus space, the Fisher information per neuron is not constant 
and therefore must be reported as an average. The fact that both of these conditions must be true leaves us with the case where we are interested only in limited regions of the stimulus space and for each neuron are concerned only with the contribution to encoding accuracy that lies within this space.

In our model, the limits to the encoding accuracy are governed by the limits to the range of the preferred stimuli. The minimum Fisher information across the finite stimulus space of interest can be increased by increasing the range of the preferred stimuli. The cost of this increase is both an increase in the number of neurons and a decrease in the average Fisher information per neuron within the stimulus space. The optimal tuning width for three dimensions is dependent on the distribution of the preferred stimuli within the stimulus space. The average Fisher information per neuron at a given tuning width will change depending on the desired value for $J_{\text {cutoff. }}$. However, the following rule is universal under the framework of our model: the encoding accuracy will improve with narrow tuning for one and two dimensions, and for higher dimensions there will be at least one optimal tuning width.

In general, when a finite number of neurons are considered to encode a finite stimulus space, there should be an optimal tuning width (in terms of encoding accuracy) for any dimension. Although our results show an infinitesimal tuning width to be optimal for one and two dimensions, for a finite number of neurons this cannot be the case, as the tuning curves will become too narrow to cover the stimulus space without gaps (Eurich \& Wilke, 2000; Wilke \& Eurich, 2002). Therefore, the variance in the encoding accuracy as a function of the tuning width is also important to consider. We have based our work on the model developed by Zhang and Sejnowski (1999), assuming independent spike firing, constant tuning widths, radial symmetric tuning curves, and neuron distributions such that the estimation errors in different dimensions are always identical and uncorrelated. The model is desirable in that it is mathematically simple and therefore useful for studying the effect of dimensionality on encoding accuracy. However, when applied in a biological setting, many other factors have been shown to influence optimal tuning widths. In addition to Fisher information and variance of encoding accuracy, an objective function for optimal tuning widths should also consider energetic constraints (Bethge, Rotermund, \& Pawelzik, 2002), heterogeneity in the tuning widths across distinct stimulus dimensions (Eurich \& Wilke, 2000), heterogeneity in the tuning widths within a stimulus dimension (Wilke \& Eurich, 2002), noise models (Wilke \& Eurich, 2002), covariance of the noise (Karbowski, 2000; Pouget, Deneve, Ducom, \& Latham, 1999; Wilke \& Eurich, 2002; Wu, Amari, \& Nakahara, 2002), nonsymmetric tuning curves (Eurich \& Wilke, 2000), decoding time and maximum firing rates (Bethge et al., 2002), hidden dimensions (Eurich \& Wilke, 2000), choice of encoding variable(s) (Eckhorn, Grusser, Kroller, Pellnitz, \& Popel, 1976), and biased estimators. 


\section{Appendix}

The steps in the integration used to derive the average Fisher information per neuron for one dimension $\left(J_{f 1 D}\right)$ are given below (The integrations for higher dimensionalities are similar):

$$
\begin{aligned}
& \overline{J_{f 1 D}}=\int_{0}^{1} d c \int_{0}^{1} J(x, c) d x \\
& \int_{0}^{1} J(x, c) d x=\int_{0}^{1} F \tau \frac{(x-c)^{2}}{\sigma^{4}} \exp \left(-\frac{(x-c)^{2}}{2 \sigma^{2}}\right) d x \\
& =F \tau \sigma^{-4} \exp \left(-\frac{c^{2}}{2 \sigma^{2}}\right) \int_{0}^{1}(x-c)^{2} \exp \left(-\frac{x^{2}}{2 \sigma^{2}}+\frac{c x}{\sigma^{2}}\right) d x \\
& =F \tau \sigma^{-4} \exp \left(-\frac{c^{2}}{2 \sigma^{2}}\right)\left(\begin{array}{l}
\int_{0}^{1} x^{2} \exp \left(-\frac{x^{2}}{2 \sigma^{2}}+\frac{c x}{\sigma^{2}}\right) d x \\
-\int_{0}^{1} 2 c x \exp \left(-\frac{x^{2}}{2 \sigma^{2}}+\frac{c x}{\sigma^{2}}\right) d x \\
+\int_{0}^{1} c^{2} \exp \left(-\frac{x^{2}}{2 \sigma^{2}}+\frac{c x}{\sigma^{2}}\right) d x
\end{array}\right) \\
& =F \tau \sigma^{-4} \exp \left(-\frac{c^{2}}{2 \sigma^{2}}\right) \\
& \times\left(\begin{array}{c}
\left.\sigma^{2} x \exp \left(-\frac{x^{2}}{2 \sigma^{2}}+\frac{c x}{\sigma^{2}}\right)\right]_{0}^{1}+c \sigma^{2} \int_{0}^{1} x \exp \left(-\frac{x^{2}}{2 \sigma^{2}}+\frac{c x}{\sigma^{2}}\right) d x \\
+\sigma^{2} \int_{0}^{1} \exp \left(-\frac{x^{2}}{2 \sigma^{2}}+\frac{c x}{\sigma^{2}}\right) d x \\
\left.-2 c \sigma^{2} \exp \left(-\frac{x^{2}}{2 \sigma^{2}}+\frac{c x}{\sigma^{2}}\right)\right]_{0}^{1}-2 c^{2} \int_{0}^{1} \exp \left(-\frac{x^{2}}{2 \sigma^{2}}+\frac{c x}{\sigma^{2}}\right) d x \\
\left.+\frac{\sigma c^{2} \sqrt{2 \pi}}{2} \exp \left(\frac{c^{2}}{2 \sigma^{2}}\right) \operatorname{erf}\left(\frac{\sqrt{2}(\mathrm{x}-\mathrm{c})}{2 \sigma}\right)\right]_{0}^{1}
\end{array}\right) \\
& =F \tau \sigma^{-4} \exp \left(-\frac{c^{2}}{2 \sigma^{2}}\right) \\
& \times\left(\begin{array}{c}
\left.\left.\sigma^{2} x \exp \left(-\frac{x^{2}}{2 \sigma^{2}}+\frac{c x}{\sigma^{2}}\right)\right]_{0}^{1}-c \sigma^{2} \exp \left(-\frac{x^{2}}{2 \sigma^{2}}+\frac{c x}{\sigma^{2}}\right)\right]_{0}^{1} \\
+c^{2} \int_{0}^{1} \exp \left(-\frac{x^{2}}{2 \sigma^{2}}+\frac{c x}{\sigma^{2}}\right) d x \\
\left.+\frac{\sigma^{3} \sqrt{2 \pi}}{2} \exp \left(\frac{c^{2}}{2 \sigma^{2}}\right) \operatorname{erf}\left(\frac{\sqrt{2}(x-c)}{2 \sigma}\right)\right]_{0}^{1} \\
\left.2 c \sigma^{2} \exp \left(-\frac{x^{2}}{2 \sigma^{2}}+\frac{c x}{\sigma^{2}}\right)\right]_{0}^{1} \\
\left.-c^{2} \sigma \sqrt{2 \pi} \exp \left(\frac{c^{2}}{2 \sigma^{2}}\right) \operatorname{erf}\left(\frac{\sqrt{2}(x-c)}{2 \sigma}\right)\right]_{0}^{1} \\
\left.+\frac{\sigma c^{2} \sqrt{2 \pi}}{2} \exp \left(\frac{c^{2}}{2 \sigma^{2}}\right) \operatorname{erf}\left(\frac{\sqrt{2}(\mathrm{x}-\mathrm{c})}{2 \sigma}\right)\right]_{0}^{1}
\end{array}\right)
\end{aligned}
$$




$$
\begin{aligned}
& =F \tau \sigma^{-2} \exp \left(-\frac{c^{2}}{2 \sigma^{2}}\right)\left((c-1) \exp \left(\frac{c}{\sigma^{2}}-\frac{1}{2 \sigma^{2}}\right)-c\right) \\
& -F \tau \frac{\sqrt{2 \pi}}{2 \sigma}\left(\operatorname{erf}\left(\frac{\sqrt{2}(c-1)}{2 \sigma}\right)-\operatorname{erf}\left(\frac{c \sqrt{2}}{2 \sigma}\right)\right) \\
& \overline{J_{f 1 D}}=F \tau \sigma^{-2} \int_{0}^{1} \exp \left(-\frac{c^{2}}{2 \sigma^{2}}\right)\left((c-1) \exp \left(\frac{c}{\sigma^{2}}-\frac{1}{2 \sigma^{2}}\right)-c\right) d c \\
& -F \tau \frac{\sqrt{2 \pi}}{2 \sigma} \int_{0}^{1}\left(\operatorname{erf}\left(\frac{\sqrt{2}(c-1)}{2 \sigma}\right)-\operatorname{erf}\left(\frac{c \sqrt{2}}{2 \sigma}\right)\right) d c \\
& =F \tau \sigma^{-2} \exp \left(-\frac{1}{2 \sigma^{2}}\right) \int_{0}^{1} \exp \left(-\frac{c^{2}}{2 \sigma^{2}}+\frac{c}{\sigma^{2}}\right)(c-1) d c \\
& -F \tau \sigma^{-2} \int_{0}^{1} c \exp \left(-\frac{c^{2}}{2 \sigma^{2}}\right) d c \\
& -F \tau \frac{\sqrt{2 \pi}}{2 \sigma} \int_{0}^{1} \operatorname{erf}\left(\frac{\sqrt{2}(c-1)}{2 \sigma}\right) d c-F \tau \frac{\sqrt{2 \pi}}{2 \sigma} \int_{0}^{1} \operatorname{erf}\left(\frac{c \sqrt{2}}{2 \sigma}\right) d c \\
& \left.=-F \tau\left(\exp \left(-\frac{1}{2 \sigma^{2}}\right) \exp \left(-\frac{c^{2}}{2 \sigma^{2}}+\frac{c}{\sigma^{2}}\right)-\exp \left(-\frac{c^{2}}{2 \sigma^{2}}\right)\right)\right]_{0}^{1} \\
& \left.-F \tau \frac{\sqrt{2 \pi}}{2 \sigma}\left(c \cdot \operatorname{gerf}\left(\frac{\sqrt{2}(c-1)}{2 \sigma}\right)\right]_{0}^{1}+\int_{0}^{1} c \frac{d}{d c} \operatorname{erf}\left(\frac{\sqrt{2}(c-1)}{2 \sigma}\right) d c\right) \\
& \left.-F \tau \frac{\sqrt{2 \pi}}{2 \sigma}\left(c \cdot g \operatorname{erf}\left(\frac{c \sqrt{2}}{2 \sigma}\right)\right]_{0}^{1}+\int_{0}^{1} c \frac{d}{d c} \operatorname{erf}\left(\frac{c \sqrt{2}}{2 \sigma}\right) d c\right) \\
& \left.=-F \tau\left(\exp \left(-\frac{1}{2 \sigma^{2}}\right) \exp \left(-\frac{c^{2}}{2 \sigma^{2}}+\frac{c}{\sigma^{2}}\right)-\exp \left(-\frac{c^{2}}{2 \sigma^{2}}\right)\right)\right]_{0}^{1} \\
& -F \tau \frac{\sqrt{2 \pi}}{2 \sigma}\left(c \cdot \operatorname{gerf}\left(\frac{\sqrt{2}(c-1)}{2 \sigma}\right)\right]_{0}^{1} \\
& \left.+\int_{0}^{1} \frac{c \sqrt{2}}{\sigma \sqrt{\pi}} \exp \left(-\frac{c^{2}}{2 \sigma^{2}}+\frac{c}{\sigma^{2}}-\frac{1}{2 \sigma^{2}}\right) d c\right) \\
& \left.-F \tau \frac{\sqrt{2 \pi}}{2 \sigma}\left(c \cdot g \operatorname{erf}\left(\frac{c \sqrt{2}}{2 \sigma}\right)\right]_{0}^{1}-\int_{0}^{1} \frac{c \sqrt{2}}{\sigma \sqrt{\pi}} \exp \left(-\frac{c^{2}}{2 \sigma^{2}}\right) d c\right) \\
& \left.=-F \tau\left(\exp \left(-\frac{1}{2 \sigma^{2}}\right) \exp \left(-\frac{c^{2}}{2 \sigma^{2}}+\frac{c}{\sigma^{2}}\right)-\exp \left(-\frac{c^{2}}{2 \sigma^{2}}\right)\right)\right]_{0}^{1} \\
& -F \tau \frac{\sqrt{2 \pi}}{2 \sigma}\left(c \cdot \operatorname{gerf}\left(\frac{\sqrt{2}(c-1)}{2 \sigma}\right)\right]_{0}^{1}+\left(\operatorname{erf}\left(\frac{\sqrt{2}(c-1)}{2 \sigma}\right)\right. \\
& \left.\left.\left.-\frac{\sigma \sqrt{2}}{\sqrt{\pi}} \exp \left(-\frac{c^{2}}{2 \sigma^{2}}+\frac{c}{\sigma^{2}}-\frac{1}{2 \sigma^{2}}\right)\right]_{0}^{1}\right)\right) \\
& \left.\left.-F \tau \frac{\sqrt{2 \pi}}{2 \sigma}\left(c \cdot g \operatorname{erf}\left(\frac{c \sqrt{2}}{2 \sigma}\right)\right]_{0}^{1}+\frac{\sigma \sqrt{2}}{\sqrt{\pi}} \exp \left(-\frac{c^{2}}{2 \sigma^{2}}\right)\right]_{0}^{1}\right) \\
& =F \tau\left(\frac{\sqrt{2 \pi}}{\sigma} \operatorname{erf}\left(\frac{\sqrt{2}}{2 \sigma}\right)+4 \exp \left(-\frac{1}{2 \sigma^{2}}\right)-4\right)
\end{aligned}
$$




\section{Acknowledgments}

We thank Shawn Martin at Sandia National Laboratories and the reviewers for their guidance in presenting this work. Support for this work was provided by Sandia National Laboratories' LDRD and Mathematics, Information, and Computational Sciences Program of the U.S. Department of Energy, and Caltech's Beckman Institute. Sandia is a multiprogram laboratory operated by Sandia Corp., a Lockheed Martin Company, for the U.S. Department of Energy's National Nuclear Security Administration.

\section{References}

Attneave, F. (1954). Some informational aspects of visual perception. Psychological Review, 61(3), 183-193.

Baldi, P., \& Heiligenberg, W. (1988). How sensory maps could enhance resolution through ordered arrangements of broadly tuned receivers. Biological Cybernetics, 59(4-5), 313-318.

Barlow, H. B. (1972). Single units and sensation: A neuron doctrine for perceptual psychology? Perception, 1(4), 371-394.

Bethge, M., Rotermund, D., \& Pawelzik, K. (2002). Optimal short-term population coding: When Fisher information fails. Neural Computation, 14(10), 2317-2351.

Cover, T. M., \& Thomas, J. A. (1991). Elements of information theory. New York: Wiley.

Eckhorn, R., Grusser, O. J., Kroller, J., Pellnitz, K., \& Popel, B. (1976). Efficiency of different neuronal codes: Information transfer calculations for three different neuronal systems. Biological Cybernetics, 22(1), 49-60.

Eurich, C. W., \& Schwegler, H. (1997). Coarse coding: Calculation of the resolution achieved by a population of large receptive field neurons. Biological Cybernetics, 76(5), 357-363.

Eurich, C. W., \& Wilke, S. D. (2000). Multidimensional encoding strategy of spiking neurons. Neural Computation, 12(7), 1519-1529.

Georgopoulos, A. P., Schwartz, A. B., \& Kettner, R. E. (1986). Neuronal population coding of movement direction. Science, 233(4771), 1416-1419.

Hinton, G. E., McClelland, J. L., \& Rumelhart, D. E. (1986). Distributed representations. In J. L. McClelland (Ed.), Parallel distributed processing (Vol. 1, pp. 77-109). Cambridge, MA: MIT Press.

Karbowski, J. (2000). Fisher information and temporal correlations for spiking neurons with stochastic dynamics. Physical Review E, 61(4 Pt. B), 4235-4252.

Knudsen, E. I., \& Konishi, M. (1978). A neural map of auditory space in the owl. Science, 200(4343), 795-797.

Kuffler, S. W. (1953). Discharge patterns and functional organization of mammalian retina. Journal of Neurophysiology, 16(1), 37-68.

Lee, B. B. (1999). Single units and sensation: A retrospect. Perception, 28(12), 14931508.

Lettvin, J. Y., Maturana, H. R., McCulloch, W. S., \& Pitts, W. H. (1959). What the frog's eye tells the frog's brain. Proceedings of the Institute of Radio Engineers (New York), 47, 1940-1951. 
Pouget, A., Deneve, S., Ducom, J. C., \& Latham, P. E. (1999). Narrow versus wide tuning curves: What's best for a population code? Neural Computation, 11(1), 85-90.

Salinas, E., \& Abbott, L. F. (1994). Vector reconstruction from firing rates. Journal of Computational Neuroscience, 1(1-2), 89-107.

Seung, H. S., \& Sompolinsky, H. (1993). Simple models for reading neuronal population codes. Proceedings of the National Academy of Sciences U S A, 90(22), 1074910753.

Snippe, H. P. (1996). Parameter extraction from population codes: A critical assessment. Neural Computation, 8(3), 511-529.

Snippe, H. P., \& Koenderink, J. J. (1992). Information in channel-coded systems: Correlated receivers. Biological Cybernetics, 67(2), 183-190.

Wilke, S. D., \& Eurich, C. W. (2002). Representational accuracy of stochastic neural populations. Neural Computation, 14(1), 155-189.

Wu, S., Amari, S., \& Nakahara, H. (2002). Population coding and decoding in a neural field: A computational study. Neural Computation, 14(5), 999-1026.

Zhang, K., \& Sejnowski, T. J. (1999). Neuronal tuning: To sharpen or broaden? Neural Computation, 11(1), 75-84.

Received September 17, 2004; accepted November 1, 2005. 